\title{
EL LAHAR TINGUIRIRICA: SU SIGNIFICADO ENTRE LOS LAHARES CHILENOS
}

\author{
THE TINGUIRIRICA LAHAR: ITS SIGNIFICANCE AMONG CHILEAN LAHARS
}

\author{
GERHARD ABELE \\ Geographisches Institut, Johannes Gütenberg-Universität \\ Mainz, Alemania
}

\begin{abstract}
The Tinguiririca lahar is an example of many similar landforms, which cover large surfaces both in andine valleys and the Chilean longitudinal depression. It is smaller than other ones in Chile, but - at the same time - better conserved, offering good conditions for genetic, kinematic and geochronologic studies, respecting volcanic mudflows.
\end{abstract}

From the genetic and kinematic point of view, the longitudinal lahar profile may be divided in two parts: a) An upper and middle accumulative area, with concave transverse profile; b) A low accumulative area; whose transverse profile is convexe. These two profiles could be explained by means of braking process in lateral parts, related to central parts, as a function of longitudinal distances from the origin.

From the geochronologic point of view, the lahar was produced between the Last Glaciation and Late Glacial times. Therefore, it is more recent than other ones.

\section{INTRODUCCION}

\subsection{ANTECEDENTES Y OBJETIVOS}

En la cordillera de San Fernando (Zona Central de Chile; $34^{\circ} 30^{\prime} \mathrm{S}$ ) el valle del río Tinguiririca y uno de sus tributarios del norte, el valle del río del Azufre, tienen un relleno de una lengua de detritos que posee una longitud de aproximadamente $55 \mathrm{~km}$ y un espesor máximo de casi $100 \mathrm{~m}$ en la parte baja y más de $200 \mathrm{~m}$ en la porte alta. A primera vista la interpretación de estos detritos como morrena en la literatura hasta ahora (LANKENAU 1958) es comprensible a causa de la ausencia de estratificación y clasificación del material.

Frente a esta interpretación hay que atender a la configuración de la parte 
inferior de $16 \mathrm{~km}$ de longitud con su superficie muy lisa, su perfil transversal convexo y la ausencia de acumulaciones glacifluviales (tipo sandur). A causa de la procedencia de esta lengua de detritos del pie del volcán Tinguiririca la interpretación como lahar (corriente de barro de origen volcánico) es convincente.

Este cuerpo de detritos en el valle Tinguiririca es un ejemplo de un gran número de cuerpos parecidos que cubren grandes superficies en los valles andinos y en la depresión longitudinal de la Zona Central chilena, por ejemplo en el valle del río Maipo (BORDE 1966), río Cachapoal (SANTANA 1971), río Teno (DAVIDSON 1974; MAC PHAIL, 1973), río Claro (ABELE, 1979; MARANGUNIC et al, 1979) y río Laja (MAC PHAIL, 1966). Como en muchos de los lahares chilenos, es más fácil reconocer y delimitar el lahar Tinguiririca en las partes bajas, es decir distales, que en las cercanías a su procedencia. Por esta causa es mejor empezar el análisis geomorfológico desde las partes bajas de las acumulaciones.

El lahar del Tinguiririca está mencionado en ABELE (1976 y 1979) y MARANGUNIC et al (1979).

En este trabajo se persigue, mediante observaciones de geomorfología comparada, llegar a establecer la importancia científica del lahar que es objeto de este estudio, en relación con otros lahares chilenos. Conjuntamente, se pretende extraer nociones genéticas, cinemáticas y geocronológicas que puedan agregarse a la teoría de estas corrientes de barro.

\subsection{METODOS}

Las investigaciones en el terreno fueron hechas en el verano de 1975/76. Pero la cartografía de las partes altas sólo fué completada en enero de 1981.

La observación se basó en el estudio de la carta 1:50.000, de fotografías aéreas y del terreno mismo. Los aspectos cinemático y genético fueron conocidos mediante el análisis de formas y de materiales, siguiendo un camino inductivo. Los aspectos geocronológicos fueron estudiados con los métodos de correlación estratigráfica.

\section{EL AREA DE ACUMULACION (fig. 1)}

\subsection{EL AREA DE ACUMULACION BAJA}

Antes de su salida de los Andes, el río Tinguiririca corre en un valle muy amplio que está cubierto de un cuerpo de detritos con gran espesor en forma de una lengua de un glaciar muy grande. La superficie de esta lengua de escombros, que baja continua y gradualmente valle abajo, es muy lisa. En el perfil transversal ella tiene una forma poco convexa con una elevación mayor en la mitad del valle. Esta es la causa por la que los ríos no se encuentran en la mitad del valle sino en sus márgenes. El río Tinguiririca corre en la depresión derecha. El río Claro, un tributario del sur, no puede desembocar en el río Tinguiririca cuando llega al valle principal, sino que está desviado en la depresión izquierda por donde corre paralelo al río Tinguiririca. Aunque la distancia entre los dos ríos es en una parte solamente $300 \mathrm{~m}$ el río Claro no puede desembocar antes del final de la lengua de detritos, después de una distancia de $16 \mathrm{~km}$ en la cual acompañaba al río Tinguiririca al otro lado del valle. Esta confluencia parece marcar el fin de los escombros (fig. 2 y 3 ). Pero también cerca de lo de sembocadura al lado derecho del ría Tinguiririca hay cortes con acumulaciones, que 


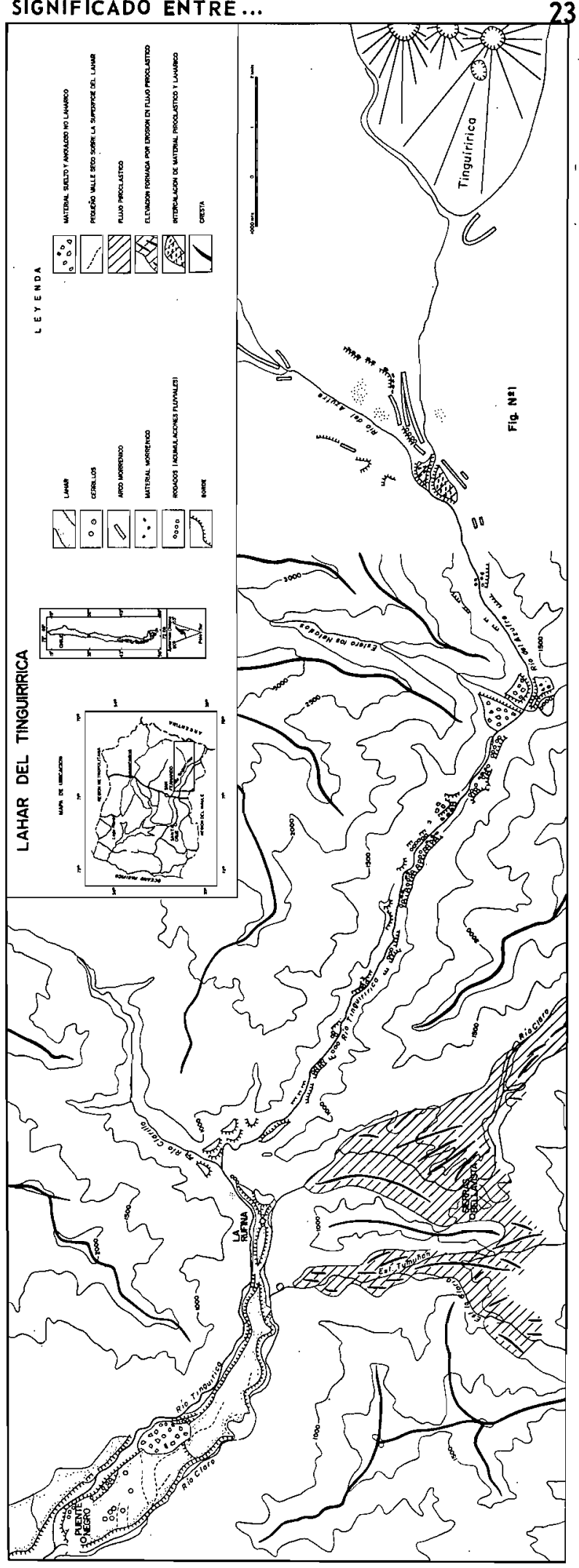




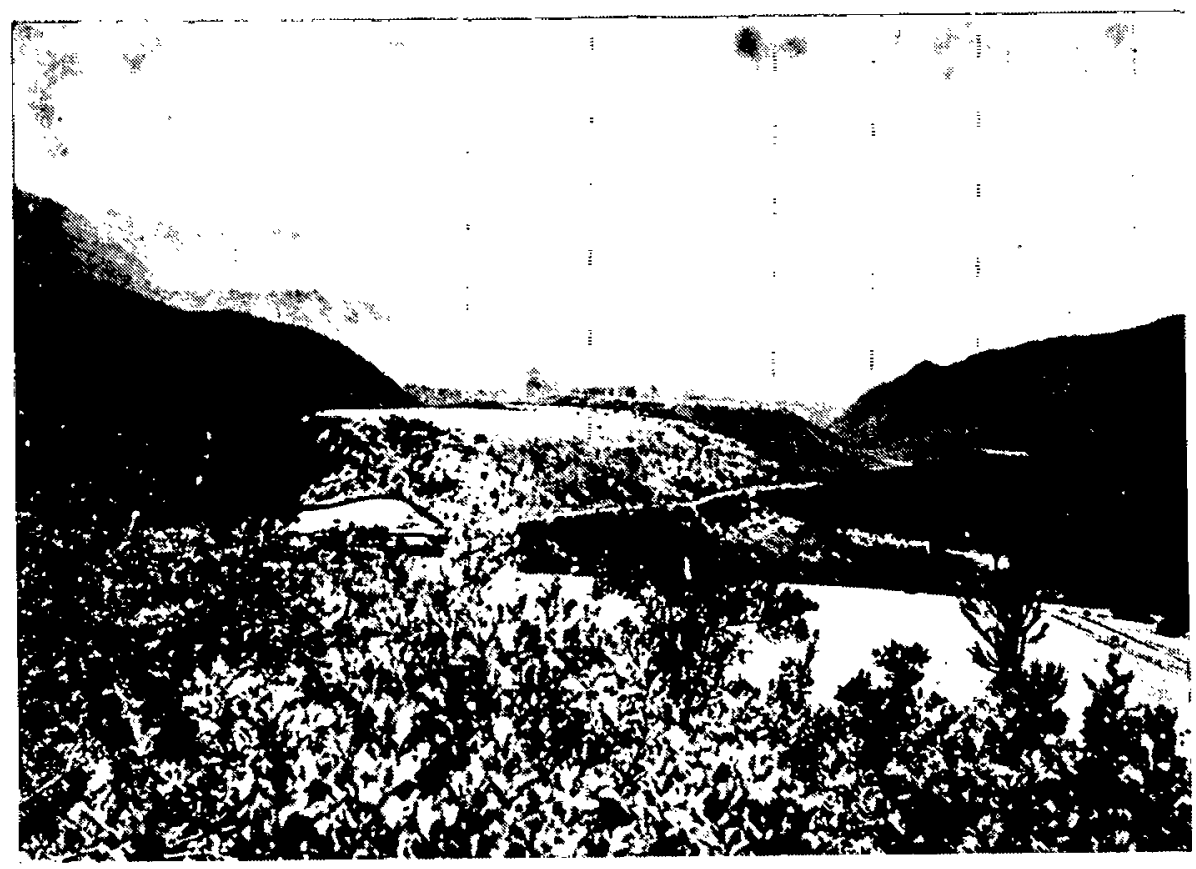

Fig. 2 Labar del Tinguiririca cerca La Rufina. Vista valle abajo. A la derecha se incidia el rio Tinguiririca a la izquierda el río Claro.



Fig. 3 Fin del labar del Tinguiririca cerca Puente Negro. Vista valle arriba. Entre los dos rios (rio Tinguiririca a la izquierda, río Claro a la derecba) se ve la lengua suavemente convexa del labar. 
indican una continuación con pequeño espesor valle abajo.

El río Tinguiririca se incide hasta más de $100 \mathrm{~m}$ y el río Claro, hasta casi $100 \mathrm{~m}$ bajo la superficie original de la lengua de detritos. Pero esto no significa que el espesor máximo sea tan grande, porque en las partes inferiores en unos lugares se ve rodados estratificados, por ejemplo al norte de Puente Negro y cerca del desvío del camino a la Sierra de Bellavista. La incisión de los dos ríos elaboró dos cañones cuyas paredes se unen en una berma muy aguda con la superficie del lahar. Por esta causa la forma positiva en la mitad del valle está acentuada. El nombre de esta forma, Isla de Briones, se puede explicar de su situación aislada entre los dos cañones.

En adición a estas formas grandes, la lengua de escombros muestra solamente pocas formas que hacen variar la condición lisa de la superficie. En este contexto se debe mencionar sobre todo las dos depresiones de aproximadamente $30 \mathrm{~m}$ en la parte superior de la Isla de Briones. En la parte baja de la lengua hay cerrillos o pequeñas elevaciones de forma redonda y una altura de hasta $5 \mathrm{~m}$. Elevaciones parecidas tiene la lengua del lahar del Teno (MAC PHAIL, 1973).

La elevación al oeste de la Loma de las Cabras, entre Las Peños y La Virgen, está constuitída de rocas sueltas y angulosas no laháricas. El lahar del Tinguiririca encontró este montón de detritos durante su curso valle abajo. EI montón formó un dique que condicionó una acumulación lahárica mayor valle arriba y una disminución abrupta del nivel de la superficie del lahar valle abajo.

Los cortes a las laderas de los dos cañones indican que la Isla de Briones está compuesta de rocas sueltas. Solamente dos elevaciones aisladas cerca de Shangri La (Puntos 724 y 778) son de roca firme. Casi todos los escombros son angulosos o subangulosos. No hay estratificación ni clasificación. El material grueso (bloques con diámetro máximo de $1 \mathrm{~m}$ y gravas se mezclan con arena y arcilla (fig. 4). Además hay muy pocos bien rodados en el cuerpo de detritos.

El extremo superior de la parte baja de la lengua de escombros se encuentra cerca de La Rufina-El Espinalillo, donde el valle del río Claro se une con el valle del río Tinguiririca. A primera vista hay tres posibilidades de continuación del lahar valle arriba: a) El valle del río Claro contiene las ignimbritas de un flujo piroclastico de gran espesor y un volumen de $3 \mathrm{~km}^{3}$ (VERGARA, 1969). b) Hay otro flujo piroclástico de cerca de $10 \mathrm{~km}$ de longitud en el valle del estero Tumuñán cuya desembocadura está solamente $2,5 \mathrm{~km}$ valle abajo de $\mathrm{La}$ Rufina. c) La lengua del lahar de la Isla de Briones se encuentra muy cerca al final de las lenguas de estos dos flujos piroclásticos. Pero no hay ninguna relación genética entre estos detritos por diferentes causas: El material de los flujos piroclásticos está consolidado y en el caso del flujo del rio Claro tiene la estructura de columnas hexagonales. En cambio la Isla de Briones tiene material no consolidado. Además en los flujos piroclásticos hay muchas incisiones de pequeños valles a pesar de la consolidación de su material. Esto indica que son mucho más viejos que las acumulaciones de la Isla de Briones que tiene una superficie fresca, alterada solamente por los dos rios principales. Es posible que los dos flujos piroclásticos hayan tenido una continuación desde el valle del estero Tumuñán al valle del río Tinguiririca y que la hayan perdido luego a causa de erosión. La superficie del final de la lengua actual del flujo piroclástico del río Claro se encuentra en un nivel mucho más alto que el nivel de la Isla de Briones. En contraste con esta diferencia de altura la superficie de la Isla de Briones se continúa sin interrupción y sin escala valle arriba hacia 
CURVAS GEOMETRICAS DE SEDIMENTOS FINOS

DEL LAHAR TINGUIRIRICA

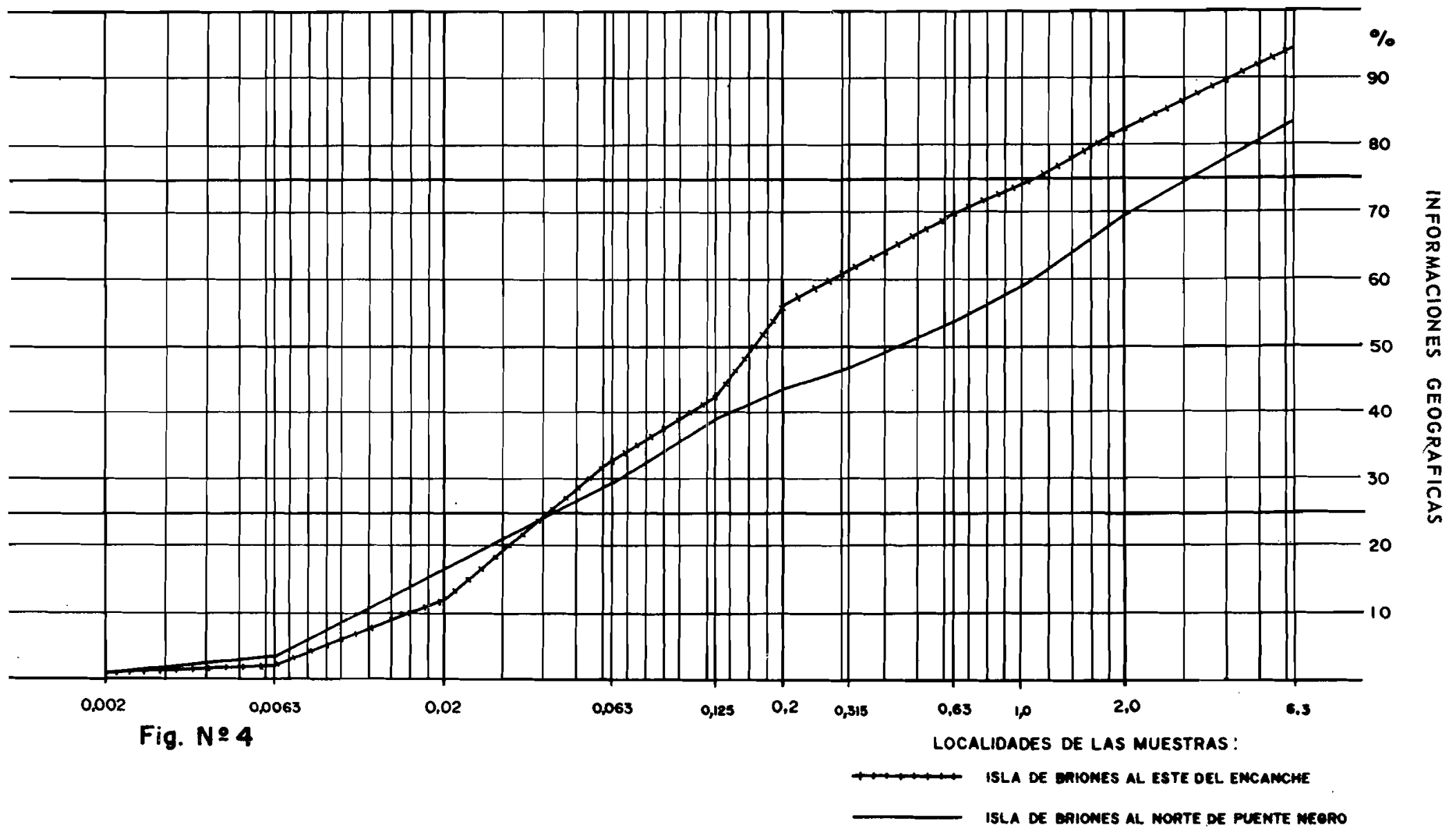


terrazas de acumulación al lado del río Tinguiririca. Además las partes superiores de estas terrazas son del mismo material anguloso que el de la lsla de Briones, sin estratificación ni clasificación. Estos hechos están en fovor de una procedencia del lahor desde el valle del Tinguiririca y no desde el río Claroo estero Tumuñán.

\subsection{EL AREA DE ACUMULACION MEDIA}

En el valle del rio Tinguiririca, se puede seguir las terrazas del lahar valle arriba hasta la desembocadura del río del Azufre. Por esta causa el límite superior del área de acumulación media debe estar en esta desembocadura. Esta parte del lahar se distingue de la parte baja en muchos aspectos: El valle es más estrecho y tiene pendientes más abruptas tanto en las vertientes como en el talweg. Los sedimentos laháricos no constituyen una forma positiva en la mitad del valle sino que forman el piso superior de terrazas de más de $100 \mathrm{~m}$ de altura en sus dos flancos. Gran parte de estas terrazas son restos del cuerpo del lahar. La conservación de partes del lahar en forma de terrazas en las dos laderas del valle es solamente posible en los casos en que el río Tinguiririca encontró en el pasado un cuerpo de detritos, cuya superficie no tenía su elevación más alta en la mitad del valle, como en el área de la Isla de Briones con su perfil transversal convexo. A causa de la inclinación de la superficie de las acumulaciones hacia la mitad del valle (perfil transversal cóncavo) el río estaba forzado a su cauce intermedio.

Solamente el piso superior de las terrazas está compuesto por el material del lahar. El piso bajo del cuerpo de las terrazas está constituído por acumulaciones de muy diferentes tipos: rodados de buena estratificación casi horizontal, rodados, arenas y arcillas de una estratificación de disposición muy caótica y rodados en una matriz fina sin estratificación. Este piso bajo de las terrazas consiste en morrenas del fondo del glaciar Tinguiririca y de sedimentos glacifluviales, en parte perturbados por el glaciar y en parte ajenos a su movimiento. Valle arriba de la desembocadura del río del Azufre no hay terrazas ni del lahar ni de los sedimentos glaciales y glacifluviales en el valle del río Tinguiririca. En cambio existen terrazas de escombros laháricos en el valle del río del Azufre.

\subsection{EL AREA DE ACUMULACION SUPERIOR}

Es difícil encontrar la continuación desde la desembocadura del rio del Azufre valle arriba. El terreno de esta desembocadura tiene un relleno de sedimentos caóticos de un movimiento de masa del valle del estero de los Helados y valle arriba hay también detritos de las laderas del valle del Azufre en gran espesor. Solamente al pie del volcán Tinguiririca, cerca de $8 \mathrm{~km}$ valle arriba de la desembocadura del río del Azufre, se ve los depósitos amarillos del lahar al lado sur del valle. Aquí los escombros laháricos, que tienen un espesor de más de $200 \mathrm{~m}$, están cubiertos de diferentes morrenas laterales de varios estadios de glaciares del valle del río del Azufre y del valle del volcán Tinguiririca.

\section{EL AREA DE PROCEDENCIA}

No hay depósitos laháricos ni en el valle del Azufre ni en el valle que baja del volcán Tinguiririca, más de $2-3 \mathrm{~km}$ aguas arriba de la confluencia de los dos rios. No obstante la dificultad de determinar la procedencia del lahar, es seguro que una corriente de barro volcánica puede solamente bajar del volcán Tinguiririca, el único volcán joven cerca del extremo superior de los escombros laháricos. 


\section{DATACION Y GENESIS DEL LAHAR}

La relación de los escombros laháricos con las morrenas y los sedimentos glacifluviales da indicaciones sobre la datación y la génesis del lahar. En el terreno de acumulación media el lahar sobreyace morrenas y sedimentos fluvioglaciales. Estos seguramente son depósitos de la última edad glacial, porque una conservación de sedimentos de las edades glaciales anteriores en un valle tan estrecho y con pendientes tan abruptas es imposible. El hecho de que el material lahárico sobreyace este material y no está acumulado en una incisión en este cuerpo glacial y glacifluvial, es una indicación de que la corriente de barro volcánica se produjio en un período en que el río Tinguiririca no había erosionado estos sedimentos. Esto significa que el lahar se formó poco tiempo después de la acumulación de los sedimentos glaciales y glacifluviales, porque normalmente incisiones en sedimentos glacifluviales en un valle con una pendiente tan grande se producen inmediatamente después del retroceso del glaciar. Esta conclusión está en buena concordancia con los hechos de la parte superior del terreno de acumulación, donde las morrenas laterales de los glaciares del valle del río del Azufre y del valle que baja del voleán Tinguiririca sobreyacen los escombros laháricos. Estas morrenas seguramente son de edad tardiglacial. Entonces, el lahar se produjo entre las acumulaciones morrénicas y glacifluviales del área de acumulación media de la última glaciación y las morrenas tardiglaciales. Con este y otros hechos mencionados más abajo se propone la siguiente secuencia de acontecimientos:

1) Acumulación de morrenas y sedimentos glacifluviales de la Ultima Glaciación en el valle del río Tinguiririca abajo de la desembocadura del río del Azufre.

2) Retroceso de los glaciares y acumulación de sedimentos glacifluviales sobre las morrenas.

3) Continuación del retroceso de los glaciares en el valle del río del Azufre y en el valle que baja del volcán Tinguiririca. Los glaciares retrocedieron al menos hasta aguas arriba de la superficie de los dos ríos. Sin este retroceso una acumulación de escombros laháricos no habría sido posible en las cercanías de esta confluencia.

4) El lahar del volcán Tinguirírica se depositó inmediatamente después de este retroceso porque las acumulaciones glacifluviales aún no estaban disectadas cuando se acumulaba el lahar en su parte media.

5) Empieza la incisión del río Tinguiririca en el cuerpo de detritos laháricos y de sedimentos glaciales y glacifluviales.

6) Avance de los glaciares tardiglaciales en el valle del río del Azufre y en el valle del voleán Tinguiririca hasta más abajo de su confluencia, formando la cuenca de Maitenes. Acumulación de morrenas laterales de diferentes es. tadios sobre el lahar.

7) Retroceso de estos glaciares.

8) Formación de diferentes acumulaciones en los valles del río del Azufre y río Tinguiririca. De arriba hacia abajo:

a) Morrenas tardiglaciales más jóvenes en el valle del río del Azufre aproximadamente $5 \mathrm{~km}$ valle arriba de las morrenas tardiglaciales mencionadas. 
b) Flujos piroclásticos y corrientes de barro que formaron abanico al pie del valle del volcán Tinguiririca.

c) Detritos de las laderas del valle del río del Azufre aguas abajo de Maitenes.

d) Movimientos de masa del valle del estero Los Helados hasta la confluencia del valle del río del Azufre con el valle del rio Tinguiririca. Estos escombros llegaron hasta el nivel actual del río Tinguiririca, lo que significa que las acumulaciones laháricas, glaciales y glacifluviales estaban disectados por el río Tinguiririca cuando se produjo este movimiento.

El contexto entre la glaciación y el lahar no solamente ofrece una buena posibilidad para datar este fenómeno, sino también para explicar este lahar. El agua necesaria para movilizar esta corriente de barro puede ser incorporada al atravesar ésta terrenos cubiertos de hielo, como en el caso del volcán Villarrica en el año 1971 (MARANGUNIC, 1974). En este sentido, se supone una génesis parecida a los lahares del Teno y Maipo (MAC PHAIL, 1973, BORDE, 1966, y WEISCHET, 1970).

\section{LAS FORMAS CONDICIONADAS POR LA CINEMATICA}

Comparando con los otros lahares en Chile, el del Tinguiririca está conservado muy bien a causa de su origen más reciente. Las incisiones de los ríos Tinguiririca y Claro, aunque profundas, no son tan anchas como para impedir una reconstrucción de la superficie original. Entonces, el lahar del Tinguiririca ofrece condiciones favorables para estudiar el movimiento de la corriente de barro. Aunque no es posible determinar la cinematica solamente considerando la configuración de los depósitos, se puede ver al menos la mecánica del efecto de freno. Considerando el perfil longitudinal es posible distinguir dos partes:

1. El área de acumulación superior y media por donde se puede reconstruir un perfil transversal cóncavo.

2. El área de acumulación baja (Isla de Briones) con su perfil transversal convexo.

Se puede explicar estos dos perfiles diferentes por el efecto de freno en las partes laterales. En las áreas de acumulación altas y medias, las partes laterales fueron depositadas cuando las partes en la mitad del valle aún estaban en movimiento. El resultado fué el perfil transversal cóncovo. En el área baja el material lateral también fué depositado antes del material intermedio. Pero en este caso las partes intermedias recibieron más material por un lapso de tiempo mayor. El resultado fué el perfil convexo. Ninguno de los dos perfiles transversales fué muy acentuado, lo que no es sorpresa en caso de una corriente de barro con poca viscosidad. El hecho de que se formaran diferencias de altura en el perfil transversal se puede atribuir a la pérdida de agua durante el efecto de freno que condicionaba una viscosidad mayor. Después de la sedimentación del lahar, los rios encontraron las depresiones cinemáticas por donde formaron sus incisiones. Esta erosión fluvial posterior es la causa de que la cavidad del perfil cóncavo y la forma positiva del perfil convexo estén más evolucionadas de lo que estaban originalmente.

Sobre casi toda el área del lahar el perfil longitudinal baja gradualmente valle abajo, pero la parte alta de la Isla de Briones posee una depresión de 
$30-40 \mathrm{~m}$ (fig. 5). Probablemente esta depresión en el perfil longitudinal se formó a causa del cambio de la velocidad del lahar, que era mayor al pie de la parte del valle más estrecha con moyor pendiente y menor, valle abajo. En este caso las dos depresiones laterales donde los ríos Claro y Tinguiririca estaban situados desde el primer momento deberían ser más profundas que esta depresión en el perfil longitudinal. Una cavidad menor de estas depresiones laterales condicionoría una confluencia de los dos ríos. En este caso su curso paralelo no sería explicable. Pero, si se supone depresiones laterales mayores, esto estaría en contradicción con la curvatura convexa muy pequeña de la lsla de Briones, de la que se puede reconstruir solamente cavidades de menos profundidad.

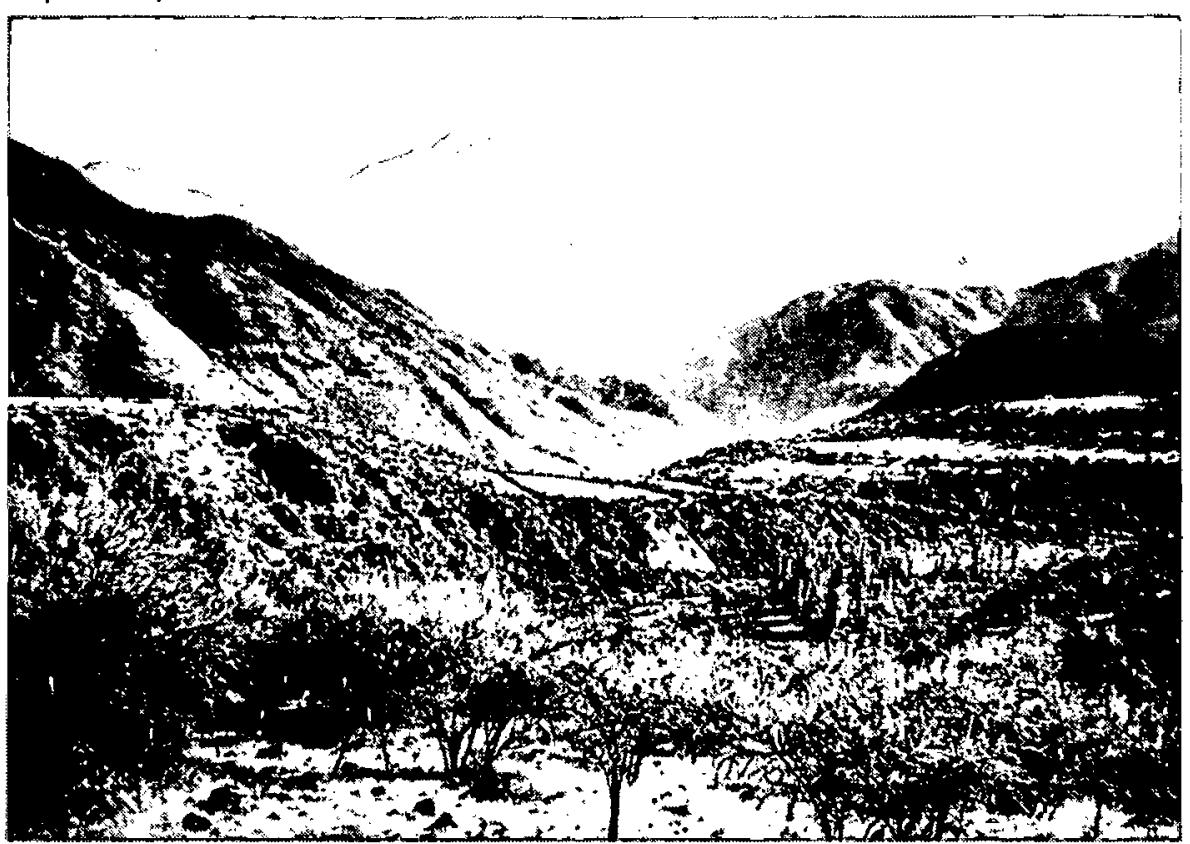

Fig. 5: Depresión longitudinal del labar cerce La Rufina.

Otra explicación para la depresión en el perfil longitudinal en la parte alta de la Isla de Briones sería su formación por erosión por el río Claro o el río Tinguiririca. Los rodados en la parte norte de la depresión son un apoyo para esta explicación. Pero si uno de los dos ríos estuviera situado en esta depresión que se extiende de una ladera del valle a ta otra, necesariamente tendría su desembocadura al otro río en este lugar. Esto significa que en este caso el curso actual de los dos ríos a los dos lados de la Isla de Briones no sería explicable. Quizás se puede explicar aquella depresión que tiene una inclinación suave al lado sur, como terraza del rio Claro, que tenía una ampliación hacia el norte y que estaba cortada después de su formación por la pendiente que baja hasta el río Tinguiririca. El conocimiento actual no permite decidir si la depresión se formó por la cinemática del lahar o por la erosión fluvial.

Otro depresión en el perfil longitudinal se encuentra $2 \mathrm{~km}$ valle abajo. Pero en este caso no estó formada por la superficie original del lahar, sino por una cresta formada por las vertientes del río Tinguiririca y del río Claro que en esta estrechez del valle tienen solamente una distancia de $300 \mathrm{~m}$.

Acá y allá la superficie suave de las partes más bajas del lahar (Isla de Briones al Este de Puente Negrol está interrumpida por cerrillos con una altura 
hasta 5 m. MAC PHAIL (1973) explica cerrillos parecidos en las partes bajas del lahar del Teno con calidad diferente del material. Según él, partes de material grueso alternan con partes de material más fino. Esto permite mencionar dos posibilidades del origen de los cerrillos. Primeramente el material grueso podría ser acumulado más temprano en un espesor mayor que el material fino y más fluído que por esta razón se ha movido hasta más lejos. El resultado de este movimiento es una superficie cuyas elevaciones más altas son los cerrillos. Pero es dificil imaginarse la génesis de estas elevaciones redondas como resultado de un movimiento diferencial. La segunda posibilidad sería la formación de los cerrillos como resultado de la deposición del lahar. Tras el escape del agua del cuerpo del lahar la superficie se rebajó más en las partes más finas que en las partes más gruesas. Aunque en este caso la formación de los cerrillos no dependería de la cinemática del lahar, esta solución tampoco permite explicar la forma aislada y redonda de los cerrillos. Un hundimiento posterior de los escombros laháricos hubiera producido formas más irregulares y además depresiones al lado de las elevaciones. Quizás ninguna de las dos soluciones de MAC PHAIL corresponde a la realidad. Cerrillos se encuentran solamente en corrientes de barro de origen volcánico y no en corrientes de barro normales. Esto quiere decir que estas elevaciones tienen un origen típicamente lahárico (escape local de vapor??). Desgraciadamente los cortes en los lahares de los ríos Teno y Tinguiririca no permiten una solución final de este problema.

\section{EL LAHAR DEL TINGUIRIRICA EN COMPARACION CON OTROS LAHARES CHILENOS}

El lahar del Tinguiririca es mucho menor que otros lahares prehistóricos en Chile. En la región de Victoria por ejemplo la depresión longitudinal esta cubierto por un abanico extenso de un gran número de lahares. Este abanico tiene su origen al pie de la Cordillera de los Andes y se extiende sobre una distancia norte-sur de más de $50 \mathrm{~km}$. Sus lahares superpuestos no solamente llegan hasta la Cordillera de la Costa (Traiguén), sino que entran en una depresión en ellay se extienden al suroeste casi hasta Chol Chol. Al contrario el lahar del Tinguirírica queda retenido en un valle de la Cordillera de los Andes. No obstante, esta gran diferencia de tamaño, este lahar es uno de los más impresionantes a causa de sus límites bien definidos y su muy buen estado de conservación. Encajonado entre las dos laderas del valle, su material está depositado en gran espesor. Los lahares que se extienden en la depresión longitudinal normalmente tienen un espesor mucho menor, a causa de la distribución lateral más ancha. Por esta causa los lahares sobrepuestos de Victoria tienen un espesor de pocos metros o decímetros. Esta acumulación de bancos laháricos se produjo durante un período extenso, porque algunos de los bancos tienen suelos que infrayacen a otros lahares (por ejemplo, cortes al lado de la Carretera Panamericana al norte del río Mininco).

En el lahar del Tinguiririca los ríos ocupan las depresiones cinemáticas de los dos lados, mientras que en la depresión longitudinal de Victoria (como en el río de Molina) siguen esos los varios cuerpos de acumulación cataclismáticos con resistencia distinta. El resultado de este hecho es una disposición radial de los ríos desde las puertas andinas, según la estructura del abanico de Victoria.

El estado de conservación de casi todos los lahares grandes en Chile es peor que el del lahar Tinguiririca. En las cuencas del río Maipo, río Cachapoal y río Laja las acumulaciones laháricas se levantan sobre los otros sedimentos solamente en sectores más o menos grandes. En otras partes están erosionadas 
- cubiertas por otros sedimentos. En los valles andinoś se encuentran los es. combros de estos lähares a lo más en pequeños restos, a tal punto que es difícil seguirlos hasta sus áreas de origen. La única excepción es el lahar del Teno que a causa de su origen más reciente tiene una conservación tan buena como el lahar del Tinguiririca. Allá hay formas cinemáticas parecidas con aquellas del Tinguiririca: existe el contraste entre el perfil transversal cóncavo en la parte proximal y convexo en la parte distal. Pero es verdad que el perfil convexo no se encuentra en los valles andinos, sino al contrario en la depresión longitudinal. Además el río Teno no corre al lado de las partes distales del lahar, sino más bien sorprendentemente sobre los escombros convexos en una pequeña depresión con un fondo más alto que el terreno en la orilla del lahar. Esta cavidad es la continuación de la depresión central del perfil cóncavo valle arriba. En las partes intraandinas el río Teno erosionó un valle amplio en esta cavidad central.

El lahar del Teno tiene su origen en el valle del río Claro que se reune con el río Teno en Los Queñes (MAC PHAIL 1973 y DAVIDSON 1974). $15 \mathrm{~km}$ al sur de Los Queñes y a más de $1.000 \mathrm{~m}$ de altitud la depresión central del lahar está interrumpida por un gran cuerpo de escombros con forma convexa; valle arriba la continuación de. la depresión central está acompañada no solamente de una terraza lateral como más abajo y como en el lahar Tinguiririca, sino de dos y en parte tres elevaciones longitudinales y paralelas que acompañan cada ladera del valle. Aunque estas elevaciones tienen el aspecto de morrenas laterales, no son de origen glacial; son formadas de escombros angulosos y parcialmente friccionados por un gran movimiento de masa. Es difícil decir si las acumulaciones en el valte del río Claro superior son parte del lahar del Teno o pertenecen a un movimiento de masa independiente y posterior. El mismo problema existe en la región del volcán Antuco, donde un lahar se extendió hasta la depresión longitudinal (MAC PHAIL, 1966) y donde escombros friccionados de un movimiento de masa se encuentran al pie de este volcán a los lados del río Laja. Las elevaciones muy altas al lado sur (Cerro Cóndor, el Puesto del Medio, los Chorrillos, punto 1360, la Cruz) y al lado norte (relleno del valle el estero el Toro (oriental), punto 1658 al lado sur de la Cordillera de Polcura, puntos 1228, 1170, 1165 y 9.58 entre el estero los Cipreses y el estero el Toro (occidental), bajan valle abajo y forman cerca de la Central Hidroeléctrica El Abanico varias elevaciones longitudinales paralelas. La cavidad central entre estas elevaciones al este de la central hidroeléctrica está rellenada de escombros formando una elevación convexa (colina de los puntos 1158 y 1197 entre las Rucas y los Robles). Estas partes bajos de los escombros han sido interpretadas como morrenas (BRUGGEN, 1942) y sedimentos laháricos (ENRIONE y VILLARROEL, 1962). En el Cerro Cóndor MAC PHAIL (1966) discute la posibilidad de un derrumbe. La diferencia entre las formas más erosionadas del lahar del Laja y las formas frescas de los escombros más valle arriba hablan en favor de dos diferentes movimientos, pero una decisión final no es posible aún.

Según estas observaciones, los terrenos superiores de los lahares del Teno y del Laja tienen un relleno de escombros de movimientos de masa, cuyas formas muestran un contraste entre el perfil transversal cóncavo en las partes superiores y el perfil transversal convexo en las partes bajas. Este cambio del perfil transversal es muy similar al que muestran los mismos lahares valle abajo. En ellos el cambio se produce en una escala horizontal mucho mayor. Por otro lado, el relieve en los escombros de los movimientos de masa más arriba es notoriamente mayor: los lahares solamente tienen terrazas laterales, los movimientos de masa más arriba producían diques laterales más altos. Además, en las partes distales los lahares muestran solamente una convexidad suave mientras que los movi- 
mientos de masa tienen una elevación central mucho mıyor. Por último el material de los movimientos de masa de más arrita parece mucho más friccionado que el material de los lahares más abajo. A causa de todos estos hechos las acumulaciones de los movimientos de masa en las partes altas de los lahares son muy parecidas a los grandes derrumbes de rocas de origen no volcánico. En este contexto se puede mencionar por ejemplo el derrumbe del Cerro Mesón Alto, cuyos escombros friccionados forman el dique del rín Yeso y de la laguna Negra (al norte del río Maipo, Prov. de Santiago) y en cuyas partes superiores hoy diques laterales en contraste con las partes inferiores, que tienen la altura mayor en la mitad del valle. En los derrumbes estas formas cinemáticas se producian cuando se movía una masa de rocas valle abajo, dejando atrás los diques como consecuencia de la fricción a las laderas del valle y acumulando la masa que se movía en la mitad del valle más abajo con perfil transversal cóncuvo. El relieve mayor de los depósitos de los derrumbes comparado con el de los lahares se explica de la viscosidad más alta de los flujos de rocas comparada con la de las corrientes de barro, que es mucho menor.

Contrariamente a los escombros del Teno y Laja, el lahar del Tinguiririca no muestra acumulaciones parecidas a cuerpos de derrumbes en sus partes altrs.

\section{CONCLUSION}

Como se menciona arriba, el tamaño del lahar del Tinguiririca es mucho menor que el de otros lahares prehistóricos en Chile. Pero, a causa de su buena conservación, este lahar ofrece condiciones favorables al estudio de génesis, datación y cinemática de las corrientes de barro volcánicas.

Debido a que los lahares son muy importantes en las condiciones geomorfológicas de varios valles y una buena parte de la depresión intermedia de Chile Central, su estudio comparado es cada vez más necesario.

Como se ve, las condiciones cinemáticas de la deposición de estas corrientes de barro volcánico varian, dependiendo del modelado original sobre el cual se depositaron. Esto produce diferencias en sus perfiles transversal y longitu dinal. En sus características interviene el efecto de freno, que es diferencial entre las partes laterales y centrales, en función de la distancia longitudinal desde el origen.

Finalmente, mediante el lahar Tinguiririca es posible conocer la generación más reciente de lahares cuaternarios, que corresponde a un interestadial antes del Tardiglacial.

\section{AGRADECIMIENTOS}

El autor agradece la colaboración del Sr. F. Gutiérrez, estudiante de la Universidad Católica de Santiago, en el trabajo de terreno del verano de 1981. La ayuda financiera por los viajes fué obtenida de la Deutsche Forschungs-gemeinschaft.

\section{REFERENCIAS}

ABELE, G. 1970. Schnelle Massenselbstbewegungen, ein dominanter morphodynamischer Faktor in den chilenischen Anden. Innsbrucker Geographische Studien. 5:21-38

ABELE, G. 1976. Vulkanisch bedingte Schlammströme in Chile zwischen Santiago und Puerto Montt. Deutscher Arbeitskreis fur Geomorphologie. 3. Sympo- 
sium, Karlsruhe 1976, Zusammenfassungen der Referate, Karlsruhe: 31 BORDE, J. 1966. Les Andes de Santiago et leur avant-pays. Bordeaux. BRUGGEN, J. 1942. El volcán Antuco y la geología glacial del valle del Laja. Santiago de Chile.

BRUGGEN, J. 1950. Fundamentos de la Geología de Chile. Ed. I. G.M. Santiago de Chile.

DAVIDSON, J. 1974. A quaternary mudflow (Lahar) down the Claro and Teno valleys from Planchon Volcano (Curicó Province, Chile). International Symposium on Volcanology. Santiago, Chile. Abstracts of papers. Santiago de Chile: 14. ENRIONE, A. \& P. VILLARROEL. 1962. Geología del área de la central hidroeléctrica el Tora (Prov. de Nuble). Escuela de Geología. Universidad de Chile. Memoria de Prueba. Santiago de Chile.

LANKENAU, E. 1958. Tinguiririca, Vulkanismus und Vereisung als formende Elemente der mittelchilenischen Hochkordillere. Petermanns Mitteilungen. Erg. H. 267. Gotho.

MAC PHA.IL 1966. El gran lahar del Laja. En: Estudios Geográficos. Santiago de Chile: 133-155

MAC PHAIL 1973. The Geomorphology of the Teno Lahar, Central Chile. Geographical. R. 63: 517-532

MARANGUNIC, C. 1974. The Lahar provoked by the eruption of the Villarrica Volcano in December of 1971. International Symposium on Volcanology, Santiago, Chile, Abstracts of Papers, Santiogo de Chile. :48.

MARANGUNIC, C., H. MORENO, \& J. VARELA. 1979. Observaciones sobre los depósitos de relleno de la presión longitudinal de Chile entre los ríos Tinguiririca y Maule. Segundo Congreso Geológico Chileno, Arica 1979, actas, tomo III : I 29.39

SANTANA, R. 1971. Les cendres volcaniques de la vallée du Cachapoal-Rapel (Chili). Cahiers de Geogr. Québec, 15 (35):315-332.

VERGARA, M. 1969. Rocas volcánicas y sedimentario-volcánicas en la latitud 34을 $30^{\prime} \mathrm{S}$. Chile. Universidad de Chile, Facultad de Ciencias Físicas y Matemáticas, Departamento de Geología, Publicación N032, Santiago de Chile.

WEISCHET, W. 1970. Chile, seine länderkundliche, Individualität und Struktur. Darmstadt. 